\title{
Un síndrome asociado a Fusarium oxysporum y a la salinidad del agua de riego que causa la muerte de plantas de tomate en Almería (Sureste de España)
}

\author{
A syndrome associated to Fusarium oxysporum and irrigation water salinity causing \\ death of tomato plants in Almería (Southeaster Spain)
}

\author{
Amalia Boix-Ruiz ${ }^{*}$, Juan Manuel Fernández-Morenate ${ }^{1}$, Miguel de Cara-García1, \\ Juan Antonio Sánchez-Garrido ${ }^{1}$, Francisco Camacho-Ferre ${ }^{1}$, Julio César Tello-Marquina ${ }^{1}$
}

\section{RESUMEN}

Durante 2002 a 2007 se evaluaron plantas de tomate cv Marmande Raf en el campo de Níjar (Almería) que mostraban el siguiente síndrome: marchitez en verde que alcanzaba a todas las hojas. Clorosis intensa del follaje terminando por secarse. Raíces, salvo excepciones, sin podredumbres y cuando estas aparecían eran localizadas. El xilema se mostraba teñido, en su totalidad, de color marrón intenso. Las plantas terminaban por morir. El análisis del xilema de 1.392 plantas exteriorizó Fusarium oxysporum en más del 59\%. Las curvas de la gravedad de síndrome evocaban a las de las micosis causadas por F. oxysporum f. sp. lycopersici o por F. oxysporum f. sp. radicis-lycopersici. La presencia del síndrome en los 17 predios de cultivo evaluados alcanzó hasta el 53,12\% de las plantas. Cuando se evaluó, la patogenicidad de 65 plantas aisladas de $F$. oxysporum ninguna de ellas expresó síntomas. En la zona muestreada el agua de riego utilizada es altamente salina. Así, en 6 pozos estudiados las concentraciones de $\mathrm{Na}^{+}$ oscilaron entre 51,48 y $205,96 \mathrm{meq} \cdot \mathrm{L}^{-1}$, las de $\mathrm{Cl}^{-}$entre 14,53 y $108,53 \mathrm{meq} \cdot \mathrm{L}^{-1}$, y la CE entre 0,55 y $10,1 \mathrm{dS} \cdot \mathrm{m}^{-1}$ y la Relación de Absorción de Sodio (SAR) entre 15,93 y 48,20. Las correlaciones calculadas para la gravedad del síndrome y la salinidad del agua de riego, en 6 invernaderos, oscilaron entre $\mathrm{R}^{2}=0,867$ para la CE, y $\mathrm{R}^{2}=0,8943$ para la concentración de $\mathrm{Cl}^{-}$. Los resultados sugieren que la salinidad del agua de riego pudo estar en la causalidad del síndrome observado, pero no explican el papel de Fusarium oxysporum en el xilema y en las raíces.

Palabras clave: monocultivo, desinfección del suelo, invernadero, sodificación.

\begin{abstract}
A syndrome associated to Fusarium oxysporum and irrigation water salinity causing death of tomato plants in Almería (Southeaster Spain).

A syndrome concerning tomato plants cv 'Marmande Raf' from "campo de Níjar" (Almería) was studied since 2002 to 2007. Involved plants showed these symptoms: early reversible wilt of the apex, followed by a general epinasty at all leaves level and no-reversible wilt. Leaves acquired intense chlorosis, until got dried. When plants were pulled up, roots didn't showed rot but rarely appeared located. The xylem was coloured in dark brown advancing in a long extension. Finally, plants died. A number of 1392 symptomatic plants from 17 different fields were analyzed. The analysis of the xylem resulted in Fusarium oxysporum isolation from more than 59\% of the plants. Progress disease curves obtained from the fields resembled to $\mathrm{F}$. oxysporum $f$. sp. lycopersici or $\mathrm{F}$. oxysporum $f$. sp. radicis-lycopersici diseases. The syndrome reached up to $53.12 \%$ of plants per field. All 65 F. oxysporum xylem-isolates that were inoculated by two different techniques didn't express any symptom on susceptible plants, under test conditions. Irrigation water is highly saline in the area. Six wells were analysed to determine salts composition. $\mathrm{Na}^{+}$concentrations varied between 51.48 and $205.96 \mathrm{meq} \cdot \mathrm{l}^{-1}$, Cl- between 14.53 and $108.53 \mathrm{meq} \cdot \mathrm{l}^{-1}$, and EC between 0.55 and $10.1 \mathrm{dS} \cdot \mathrm{m}^{-1}$ and RAS between 15.925 and 48.2041. Disease severity and water salinity from 6 wells showed a high correlation for $E C\left(R^{2}=0.867\right)$, and $\mathrm{Cl}^{-}$concentration $\left(\mathrm{R}^{2}=0.8943\right)$. Results suggest that water salinity can be implicated in the syndrome, but cannot explain the role of Fusarium oxysporum in the xylem and roots of diseased plants.
\end{abstract}

Key words: monoculture, soil disinfection, greenhouse, sodification.

\footnotetext{
1 Grupo de Investigación AGR-200 "Producción Vegetal en Sistemas de Cultivo Mediterráneos”. Universidad de Almería. Cañada de San Urbano s/n 04120 Almería. España.

* Autor para correspondencia: amalia6i@yahoo.es
}

Fecha de Recepción: 24 Abril, 2014.

Fecha de Aceptación: 7 Mayo, 2014. 


\section{Introducción}

El cultivo de tomate (Solanum Lycopersicum L) en la provincia de Almería ocupa una extensión de entre 8.000 a 10.000 ha en la última década, constituyéndose como una de las especies hortícolas preponderantes (Delegación de Agricultura en Almería de la Junta de Andalucía).

El trabajo que presentamos tiene su origen en diversos parajes de la comarca de Níjar, en predios donde se cultiva "tomate sabor" bajo invernadero y bajo malla (en adelante llamaremos invernadero a ambos tipos de estructura) y donde la especialización en el cv Marmande Raf hace que se obtenga de modo continuado una gran cotización de este producto en el mercado. Para el propósito de la investigación realizada es conveniente tener presente el historial de los campos donde se desarrolló la investigación. Hasta hace 65 años el cultivo del tomate se hacía al aire libre de manera generalizada. Terminada la cosecha el ganado vacuno aprovechaba parte de los restos de la misma para su alimentación. Posteriormente se daba una labor con arado de vertedera, debido a que los suelos no estaban arenados, y se sembraban cereales, generalmente maíz, para cultivar de nuevo tomate, realizando un aporte de estiércol al suelo, previo a este cultivo. Los agricultores realizaban su propio semillero y la selección de las semillas, normalmente de cultivares tipo Marmande. Fue en la década de los 60 del siglo XX cuando empezaron a realizarse los cultivos en suelos arenados y se inició la construcción de invernaderos y abrigos de malla para cultivar tomate. El agua de riego utilizada en la zona procede de pozos de una profundidad comprendida entre 11 y $25 \mathrm{~m}$, caracterizada por una elevada conductividad eléctrica, y en no pocos casos con alto contenido en boro (Segura Visiedo, 2005). En la actualidad, en un año agrícola al cultivo del tomate le precede un cultivo de melón o sandía.

La producción del tomate (en estas condiciones de salinidad) se hace para obtener una calidad gustativa muy característica y apreciada en los mercados, donde la sal incrementa considerablemente el contenido en azúcares (Cuartero et al., 1995), lo que se consigue con ciertos tipos de cultivares.

El trabajo fue realizado entre 2002 y 2007 en 17 predios (invernados o bajo mallas) y parece conveniente especificar el significado que se da al término síndrome en el texto. El diccionario de la Real Academia Española de la lengua, establece, entre otros, el siguiente significado: conjunto de fenómenos que caracterizan una situación determinada. Y es preciso tenerlo presente para no confundirlo con el que usualmente se le da para recoger el conjunto de síntomas de las enfermedades parasitarias, máxime cuando la presencia de Fusarium oxysporum (que ha desarrollado dos especializaciones patógenas sobre tomate) es protagonista de todos los experimentos que se presentan y discuten en el texto.

En este contexto se plantea el objetivo de relacionar la presencia del hongo asociado a una secuencia de síntomas y establecer una previsible relación con la salinidad del agua de riego.

\section{Materiales y Métodos}

\section{Muestreos realizados y su ubicación. Muestreos durante las campañas 2002/2003, 2003/2004 y 2004/2005}

Se realizaron en una explotación situada en Campillo de Gata (Níjar). Cultivo bajo invernadero, suelo arenado y una superficie de $18.842 \mathrm{~m}^{2}$, el cultivar fue Marmande Raf, riego por goteo. Monocultivo desde 1986. Suelos desinfectados anualmente con diversos fumigantes (1,3-dicloropropeno, 1,3-dicloropropeno + cloropicrina, metam-sodio). Agua para riego procedente de pozo. El conteo de plantas manifestando el síndrome se realizó sobre la totalidad de las plantas de la explotación (Segura Visiedo, 2005). Las evaluaciones se hicieron a lo largo del cultivo. Se codifica como Inv 17 (Tabla 1).

\section{Muestreos realizados en la campaña 2006/2007}

Se muestrearon un total de 16 invernaderos, cuyas características más destacadas, en lo que concierne a este trabajo, se resumen en la Tabla 1. Todos los invernaderos estaban situados en el término municipal de Níjar en los siguientes parajes: Los Rubiales, Campillo de Gata, Mazarulleque, Llano del Rocío, Llano Pujaire - La Loma y Las Huertas. 6,19 ha fueron evaluadas para computar el número de plantas muertas y/o con el síndrome descrito en el apartado siguiente. La evaluación se hizo sobre el total de las plantas en cada invernadero. 
Tabla 1. Características de los invernaderos muestreados durante la campaña 2006-2007.

\begin{tabular}{|c|c|c|c|c|c|}
\hline $\begin{array}{l}\text { Código } \\
\text { invernadero }\end{array}$ & $\begin{array}{l}\text { Superficie } \\
\qquad\left(\mathrm{m}^{2}\right)\end{array}$ & Tipo de suelo & $\begin{array}{l}\text { Antigüedad del cultivo } \\
\text { (años) }\end{array}$ & $\begin{array}{l}\text { Variedades de } \\
\text { tomate cultivadas }\end{array}$ & $\begin{array}{c}\text { Desinfección previa al } \\
\text { suelo }\end{array}$ \\
\hline Inv.1 & 6.300 & arenado & $>10$ monocultivo & Marmande Raf & metam-sodio \\
\hline Inv. 2 & 1.890 & arenado & 2 monocultivo & Marmande Raf & cloropicrina \\
\hline Inv. 3 & 4.023 & arenado & $>10$ monocultivo & Marmande Raf & no \\
\hline Inv.4 & 2.365 & arenado & $>10$ monocultivo & Marmande Raf & no \\
\hline Inv. 5 & 2.270 & arenado & 3 monocultivo & Marmande Raf & no \\
\hline Inv.6 & 3.700 & arenado & nueva plantación & Marmande Raf & no \\
\hline Inv.7 & 8.300 & arenado & $>5$ monocultivo & Marmande Raf & metam-sodio \\
\hline Inv. 8 & 8.000 & arenado & $>5$ monocultivo & Marmande Raf & metam-sodio \\
\hline Inv.9 & 13.200 & arenado & 2 monocultivo & Marmande Raf & no \\
\hline Inv.10 & 16.180 & arenado & 1er cultivo & Delizia F1 & no \\
\hline Inv.11 & 2.500 & arenado & 2-3 monocultivo & Delizia F1 & no \\
\hline Inv.12 & 2.000 & arenado & 2-3 monocultivo & Marmande Raf & no \\
\hline Inv.13 & 4.500 & arenado & 2-3 monocultivo & Marmande Raf & no \\
\hline Inv.14 & 2.200 & arenado & 2-3 monocultivo & Marmande Raf & no \\
\hline Inv.15 & 6.500 & arenado & 2-3 monocultivo & Marmande Raf & no \\
\hline Inv.16 & 7.000 & arenado & 2-3 monocultivo & Marmande Raf & no \\
\hline
\end{tabular}

\section{Análisis fitopatológicos de plantas. Número de plantas analizadas y síndrome observado}

En las campañas 2003/2004 y 2004/2005 se analizaron 815 plantas. En la campaña 2006/2007 se analizaron 577 plantas. Las muestras de plantas para su análisis fueron tomadas en diferentes muestreos, los que oscilaron entre 8 y 11 , repartidos a lo largo de la duración del cultivo.

Las plantas muestreadas para su análisis en el laboratorio y las evaluadas en el campo presentaron el siguiente síndrome: marchitez en verde reversible de las copas, fueron los primeros signos. Posteriormente la epinastia alcanzaba todas las hojas y la marchitez se hacía irreversible. Las hojas adquirían una clorosis intensa, terminando por secarse. $\mathrm{Al}$ arrancar las plantas, las raíces -salvo excepciones- no presentaban podredumbres, y cuando las mostraron eran parciales y no generalizadas. El xilema aparecía teñido, en gran parte de su extensión, de color marrón intenso. Era común el rehundimiento del tallo en el suelo con objeto de provocar la emisión de raíces. Raíces que eran de corta longitud y no eran capaces de mejorar el estado de las plantas. Las plantas terminaban por morir. Las plantaciones se realizaron en los invernaderos en diferentes fechas, entre agosto y diciembre de cada campaña considerada. Los primeros síntomas se expresaron a partir de enero.
Los análisis de plantas y purificación de aislados para su identificación se hicieron de la siguiente manera: para los análisis del xilema se siguió la técnica indicada por Tello Marquina y Lacasa Plasencia (1990). En esencia, trozos de tallos eran lavados minuciosamente con agua del grifo. Después del secado se flameaban con alcohol y se sembraban en PDA (Agar Patata Dextrosa) rodajas del tallo de aproximadamente $1 \mathrm{~cm}$ de espesor cortadas con tijeras flameadas con alcohol. La incubación se realizaba en la bancada del laboratorio (luz solar y temperaturas que oscilaron entre $19^{\circ} \mathrm{C}$ y $24^{\circ} \mathrm{C}$ ). Del micelio crecido sobre el xilema de cada rodaja se tomaban, con aguja enmangada flameada en alcohol, unas porciones y se transferían a PDA, para su posterior identificación. El análisis de las podredumbres de las raíces se hizo lavando estas minuciosamente con agua del grifo y dejándolas secar. Posteriormente se sembraron trozos de $1 \mathrm{~cm}^{2}$ en medio selectivo para Fusarium (Komada, 1975). Cuando el micelio había crecido sobre el medio se transfirió una porción a PDA con objeto de proceder a su inoculación e identificación posteriores. La tierra rizosférica que acompañaba a las plantas fue analizada mediante trampas vegetales para detectar la presencia de Phytophthora según técnica de Tello et al. (1991). El análisis se hizo para todas las plantas, utilizando como "trampas" para tal fin, pétalos inmaduros de clavel.

La identificación de Fusarium se hizo siguiendo el manual de Nelson et al. (1983). Se utilizó, como 
allí se indica, un medio a base de agar con cloruro potásico y trozos de hojas de clavel desinfectadas. La identificación se completó con el atlas de Gerlach y Nirenberg (1982).

\section{Inoculaciones}

Se inocularon 65 aislados de Fusarium oxysporum recogidas entre 2002 y 2007 . De ellos 4 procedían de las raíces y 61 del xilema. Las inoculaciones fueron repetidas 2 veces en el tiempo, inoculando 10 plantas por aislado en cada repetición y técnica de inoculación. Fueron utilizadas dos técnicas de inoculación:

1. Por riego al sustrato de una suspensión en agua estéril de propágulos del aislado, hasta obtener una concentración del orden de $10^{5} \mathrm{UFC} \cdot \mathrm{mL}^{-1}$. Dicha inoculación se realizó sobre plantas de tomate susceptible a las fusariosis cv San Pedro cuando tenían una hoja verdadera. El sustrato utilizado fue vermiculita desinfectada en autoclave ( 1 hora, $121^{\circ} \mathrm{C}$ ). Las semillas previamente desinfectadas por inmersión en lejía comercial ( $30-40 \mathrm{~g} \mathrm{Cl}$ activo $\left.\cdot \mathrm{L}^{-1}\right)$ durante 30 minutos y posterior aclarado con agua. $\mathrm{La}$ siembra se realizó sobre vermiculita contenida en macetas de $1 \mathrm{~L}$ de capacidad. En cada maceta se sembraron 5 semillas que dieron lugar a 5 plantas. Como cepa de referencia se utilizó un aislado de Fusarium oxysporum f. sp. radicislycopersici y otro de $F$. o. f. sp. lycopersici patotipo 1 (cepas depositadas en el Departamento de Agronomía de la Universidad de Almería).

2. Por inmersión de raíces. Las plántulas de tomate cv San Pedro fueron arrancadas del sustrato donde crecieron (vermiculita desinfectada) cuando tenían tres hojas verdaderas. Las raíces fueron sumergidas en una suspensión de propágulos en agua estéril del aislado correspondiente $\left(10^{5} \mathrm{UFC} \cdot \mathrm{mL}^{-1}\right)$ durante 30 minutos. Posteriormente fueron trasplantadas a macetas de $1 \mathrm{~L}$, a razón de 5 plantas por maceta crecidas en sustrato vermiculita desinfectada como se indicó anteriormente.

Todas las inoculaciones se hicieron en una cámara de ambiente controlado. Fotoperiodo de 16 horas de luz al día. Luminosidad de 12.000 lux al nivel de las plantas. Temperaturas oscilando entre $20^{\circ} \mathrm{C}$ y $23^{\circ} \mathrm{C}$, y entre $23^{\circ} \mathrm{C}$ a $30^{\circ} \mathrm{C}$ en la repetición.
La diferencia entre temperaturas obedeció a que la expresión de $F$. $o$. f. sp. radicis-lycopersici ocurre mejor en la primera gama, mientras que $F$. $o$. f. sp. lycopersici lo hace más nítidamente a temperaturas más elevadas. Las macetas se regaron a demanda durante los ensayos con una solución de abono cristalino 15-15-15, a razón de $1 \mathrm{~g} \cdot \mathrm{L}^{-1}$.

\section{Análisis de agua de riego}

Se muestreó el agua de 6 pozos con los que se regaban 8 de los invernaderos inventariados en la Tabla 1. La relación de dichos invernaderos con los pozos se recoge en la Tabla 2.

Los análisis se orientaron para obtener los datos correspondientes a conductividad eléctrica (CE), $\mathrm{pH}$, cationes $\left(\mathrm{Ca}^{+2}, \mathrm{Mg}^{+2}, \mathrm{Na}^{+}, \mathrm{K}^{+}\right.$y B $)$y los aniones $\left(\mathrm{CO}_{3}^{-2}, \mathrm{Cl}^{-}, \mathrm{SO}_{4}^{-2}\right.$ y $\left.\mathrm{NO}_{3}^{-}\right)$. A partir de estos datos se calculó, para cada pozo, la Ratio de Adsorción de Sodio (SAR).

\section{Técnicas estadísticas}

Se utilizó el programa informático STATGRAPHICS Plus de Windows (versión 5.1). La transformación de los datos en el caso de los valores expresados en porcentajes fue mediante arcoseno (valor/100).

\section{Resultados y Discusión}

\section{Resultados sobre las observaciones en campo y de los análisis de plantas}

Los resultados analíticos se resumen en la Tabla 3. El síndrome anteriormente descrito se repitió en todos los invernaderos a lo largo del tiempo de observación y análisis. El cuadro de

Tabla II. Códigos de pozos de los que se analizó el agua e invernaderos que fueron regados durante el tiempo de observación.

\begin{tabular}{cc}
\hline Pozo & Invernadero \\
\hline Pozo 1 & Inv.1 \\
Pozo 2 & Inv.7 \\
Pozo 3 & Inv.8 \\
Pozo 4 & Inv.4 \\
Pozo 5 & Inv. 2, Inv.3, Inv.4 \\
Pozo 6 & Inv.6 \\
\hline
\end{tabular}


Tabla 3. Aislamiento de Fusarium oxysporum en el xilema y en las raíces de plantas de los invernaderos muestreados.

\begin{tabular}{lccc}
\hline \multirow{2}{*}{$\begin{array}{l}\text { Año de } \\
\text { muestreo }\end{array}$} & \multicolumn{2}{c}{$\begin{array}{c}\text { Presencia de } F \text {. oxysporum } \\
\text { (se expresa en porcentaje sobre el total de plantas) }\end{array}$} \\
\hline $2002-2005$ & $N^{\circ}$ total de plantas analizadas & xilema & raíz \\
\cline { 2 - 3 } $2006-2007$ & 815 & 58,67 & - \\
\hline
\end{tabular}

signos se asemejaba al que se ha descrito para las dos fusariosis del tomate causadas por Fusarium oxysporum f. sp. lycopersici y para $F$. $o$. f. sp. radicis-lycopersici (Tello Marquina, 1984).

Teniendo en cuenta que se analizaron plantas sintomáticas, el porcentaje de presencia positiva de F. oxysporum no alcanza a la totalidad. Este hecho podría contravenir el primer postulado de KochPasteur para establecer la causalidad de los hongos aislados respecto del síndrome. Sin embargo, Tello Marquina y Lacasa Plasencia (1990) presentaron resultados comparables a los mostrados aquí cuando estudiaron la importancia de las fusariosis vasculares del tomate y del clavel. En su trabajo realizaron una investigación precisa sobre este punto y concluyeron que no siempre la presencia de necrosis vascular producida por Fusarium oxysporum permitía el aislamiento del hongo en el laboratorio. La presencia de $F$. oxysporum asociado a las raíces podría sugerir que estuvo presente $F$. oxysporum f. sp. radicislycopersici, pero los síntomas no correspondían con los descritos para la enfermedad que causa (Tello Marquina y Lacasa Plasencia, 1990).

Es importante señalar que no se aisló Verticillium dahliae, también agente causal de enfermedades vasculares y que tampoco fue detectada la presencia de ninguna especie de Phytophthora ni en el material vegetal ni de la tierra rizosférica. Por lo tanto, ateniéndose a los resultados y su comentario podría admitirse como válido el primer postulado Koch-Pasteur y asignar una relación a $F$. oxysporum con el síndrome.

\section{Resultados sobre la incidencia y expresión a lo largo del tiempo del síndrome en los invernaderos}

Durante las campañas comprendidas entre 2002 y 2005 las plantas con el síndrome (incluyendo las que murieron) entre enero y junio de cada año fueron para la campaña 2002/2003 el 26,66\%, y para 2004/2005,
28,83\%. Durante la campaña 2006/2007, en los 16 invernaderos, las plantas afectadas oscilaron entre el $0,45 \%$ y el $53,12 \%$. En todos los invernaderos se expresó el síndrome, excepto en el codificado como Inv13. El número de invernaderos donde el porcentaje de plantas afectadas fue superior al $15 \%$ fue de 8 (50\% del total).

Los porcentajes son próximos a los obtenidos por Tello Marquina (1984) para la evaluación de la fusariosis vascular y la verticiliosis en los tomatales de Murcia. La expresión temporal del síndrome se presenta para los invernaderos codificados como Inv1, Inv2, Inv3, Inv4, Inv5, Inv6, Inv8 en la Figura 1, para los cuales se evaluó la salinidad del agua de riego. En el Inv7 no fue posible la evaluación regular, por lo que pese a estar presente el síndrome no se representa.

La Figura 1 muestra tanto en la curva como en el ajuste de esta, para cada invernadero, una tendencia creciente que evoca los presentados por Tello Marquina (1984) para los cultivos de tomate de Águilas y Mazarrón (Murcia), que reflejan la expresión a lo largo del tiempo de muestreo de la fusariosis vascular y la verticiliosis en los cultivos de tomate. Las temperaturas de la zona estudiada son comparables a las que dicho autor señala para las comarcas murcianas. En este contexto y según el régimen térmico, debería haberse expresado Verticillium dahliae si hubiese estado en el patosistema y no solo Fusarium oxysporum (Tello Marquina, 1984).

Los datos obtenidos sugieren que la fusariosis vascular era la enfermedad presente en la zona. Sin embargo, algunos datos permitían expresar dudas razonables sobre la especulación anterior. Así, la aplicación de desinfectantes del suelo antes de plantar no mostró, aparentemente, efectos apreciables. Véase a tal efecto en la Tabla 1, como Inv1 (desinfección con metam-sodio), Inv2 (cloropicrina) no hicieron remitir la expresión del síndrome. Además, las variedades cultivadas tipo Marmande mayoritariamente no 
Inv1.

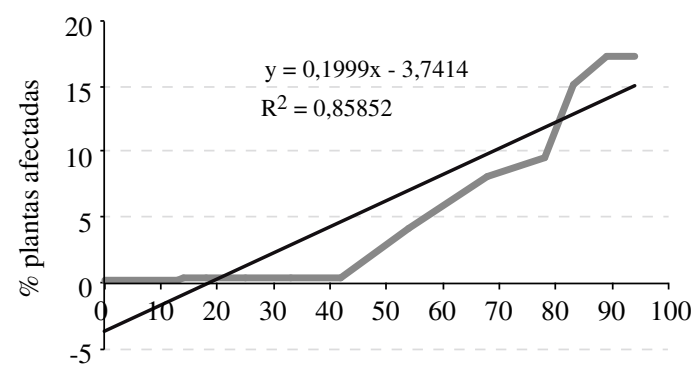

Días transcurridos desde el 15 de febrero

Inv3.

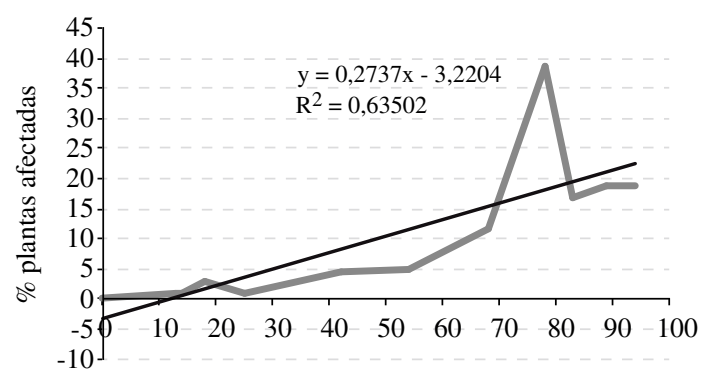

Días transcurridos desde el 15 de febrero

Inv5.

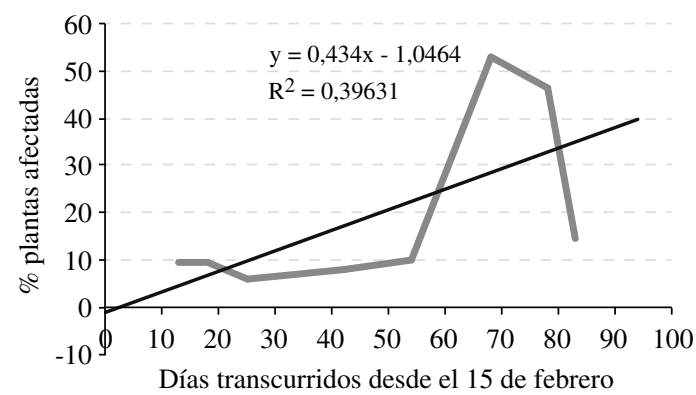

Días transcurridos desde el 15 de febrero
Inv2.

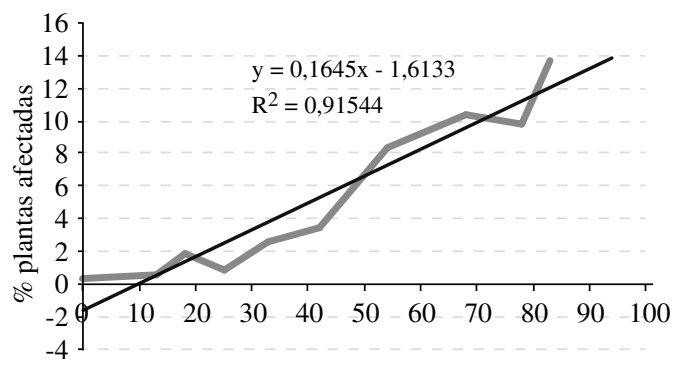

Días transcurridos desde el 15 de febrero

Inv4.

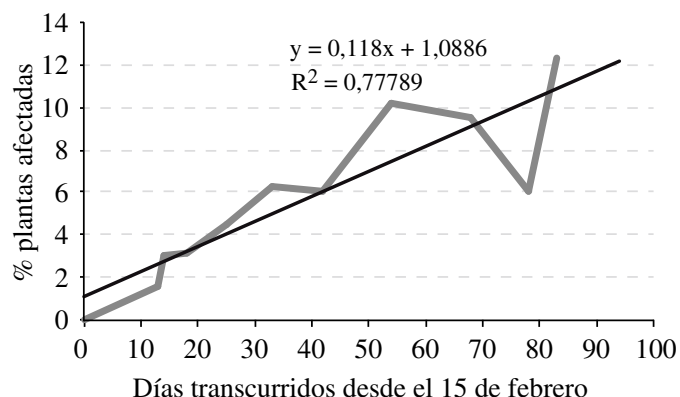

Inv6.

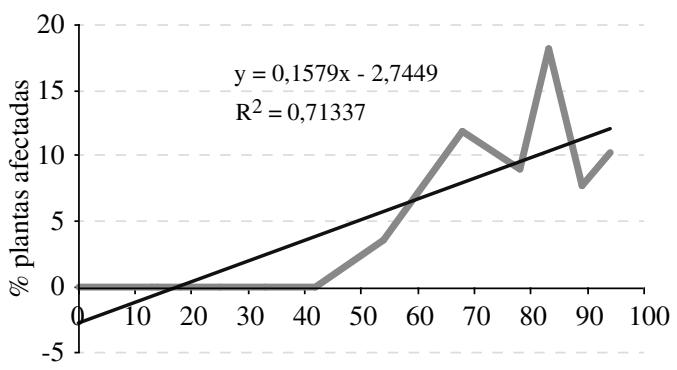

Días transcurridos desde el 15 de febrero

Inv8.

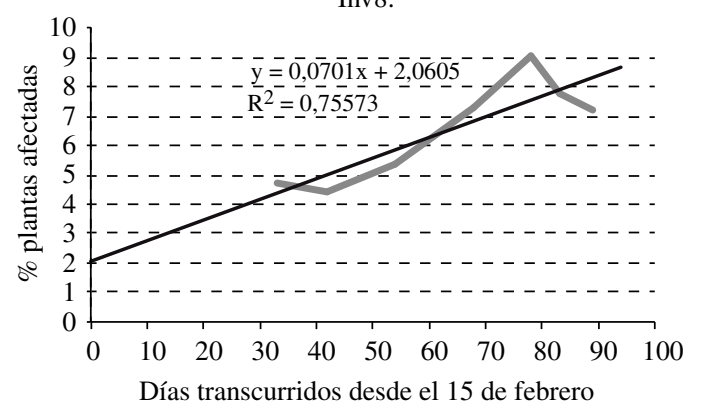

Figura 1: Evaluación del síndrome en los invernaderos regados con agua salina. A: inv. 1; B: inv. 2; C: inv. 3; D: inv. 4; E: inv. 5; F: inv. 6; G: inv. 8; Inc\%: Porcentaje de incidencia. 
representaban una barrera para la expresión de la micosis, porque no son resistentes a $F$. oxysporum f. sp. lycopersici y a $F$. oxysporum f. sp. radicislycopersici (Tello Marquina, 1984).

\section{Resultados sobre la identificación morfológica y patogénica de $\boldsymbol{F}$. oxysporum aislados}

Sesenta y cinco aislados fueron seleccionados de todos los invernaderos. La identificación morfológica los agrupó a todos bajo la especie Fusarium oxysporum. Las inoculaciones para los 65 aislados, utilizando el $c v$ San Pedro, y aplicando dos técnicas de inoculación, no permitieron que los aislados expresasen ningún tipo de patogenicidad. Sin embargo, los aislados tomados como testigos correspondientes a $F$. oxysporum f. sp. radicislycopersici y F. oxysporum f. sp. lycopersici (raza 1) sí expresaron netamente su patogenicidad. Lo que sirve de contraste para sugerir que los aislados estudiados no fueron patógenos en las condiciones de ensayo.

Los resultados presentados hasta el presente no permitían establecer la causalidad del síndrome observado a lo largo de los años. Sugerían que el conjunto de síntomas no eran una respuesta a una enfermedad parasitaria. Sin embargo, al introducir este trabajo se hacía referencia a la salinidad del agua de riego utilizada, y en la Tabla 1 se precisaban los años de monocultivo en cada invernadero. Teniendo presente este aspecto del manejo, parecería tener sentido plantearse si el agua de riego podría estar en el origen del síndrome. En este sentido, Pellicer Botia et al. (1998) describían un síndrome en los suelos sodificados cultivados con tomate de la siguiente manera: En suelo sodificado las plantas presentaban el xilema oscurecido hasta casi ennegrecerse. La planta amarillea, detiene su crecimiento y vegeta sin apenas producción. En casos de salinidad aguda la necrosis del xilema se acompaña de otra epidérmica que se remonta hasta $40 \mathrm{~cm}$ sobre el suelo. En estas situaciones el raquitismo de las plantas se acompaña de epinastias más o menos permanentes. Entre la bibliografía consultada esta descripción es la que más se aproxima al síndrome descrito anteriormente.

Los análisis de agua de riego de 6 pozos (Tabla 2), que abastecían a los invernaderos codificados como Inv1, Inv2, Inv3, Inv4, Inv5, Inv6, Inv7 e Inv8, se presentan en las Tablas 4, 5 y 6.
En las Tablas se puede comprobar que no se cumplen los estándares recomendados para aguas de riego por Ayers y Wescot (1985). Los análisis sugieren que en todos los invernaderos existe riesgo importante de salinización, excepto en los invernaderos regados por el agua del pozo 3 (Inv7). Sin embargo en ese caso el elevado SAR es indicativo de un peligro de sodificación, pese a la baja conductividad eléctrica.

La concentración de cloruros (Tabla 5) es muy elevada en todos los pozos, sobrepasando las 10 meq. $\mathrm{L}^{-1}$ que se consideran como umbral límite para el cultivo del tomate (Porta et al., 2003). La elevada presencia de boro en los pozos 4 y 5 podrían estar presentando riesgos altos de toxicidad. Riesgo que es menor para los pozos 3 y 6. Según Porta et al. (2003), el cultivo del tomate es moderadamente tolerante al boro hasta niveles de $2 \mathrm{mg} \cdot \mathrm{L}^{-1}$. Otras apreciaciones sobre la calidad de las aguas utilizadas para el riego recaen sobre los nitratos. Los pozos 1, 4 y 5 superan con creces los $50 \mathrm{mg} \cdot \mathrm{L}^{-1}$ que marca como límite la normativa española y europea en el Real Decreto 261/1996 (boletín oficial del Estado, 16 de febrero de 1996) sobre la protección de aguas contra la contaminación originada por nitratos procedentes de fuentes agrarias.

\section{Relación entre la calidad del agua y la incidencia del síndrome en los invernaderos}

La comparación de cuatro parámetros, que de manera clásica se utilizan para evaluar la salinidad del agua de riego (CE, SAR, concentración de boro, concentración de cloruros), con la incidencia de la enfermedad expresada en porcentaje de plantas muertas y/o enfermas, se ha representado en las Figuras 3, 4 y 5 . En la Figura 2 puede apreciarse una elevada correlación entre la gravedad del síndrome y la conductividad eléctrica. En la Figura 3 se muestra una correlación elevada entre el SAR y la incidencia de la enfermedad si se exceptúa el pozo 3 que riega el invernadero codificado como Inv8. Aceptando la excepción de dicha medida, la correlación es alta $\left(\mathrm{R}^{2}=0,8644\right)$ para la ecuación de la recta (porcentaje de incidencia) Inc $\%=0,3190($ SAR $)+6,9078$. Para hallar una explicación habría que tener en cuenta la menor $\mathrm{CE}=0,55 \mathrm{dS} \cdot \mathrm{m}^{-1}$ (Figura 2), aun presentando un SAR muy elevado, y que en este caso la relación entre los cationes $\mathrm{Na}^{+}, \mathrm{Ca}^{++}$y $\mathrm{Mg}^{++}$es alta debido a la menor presencia de $\mathrm{Ca}^{++} \mathrm{y} \mathrm{Mg}^{++}$, lo que puede 


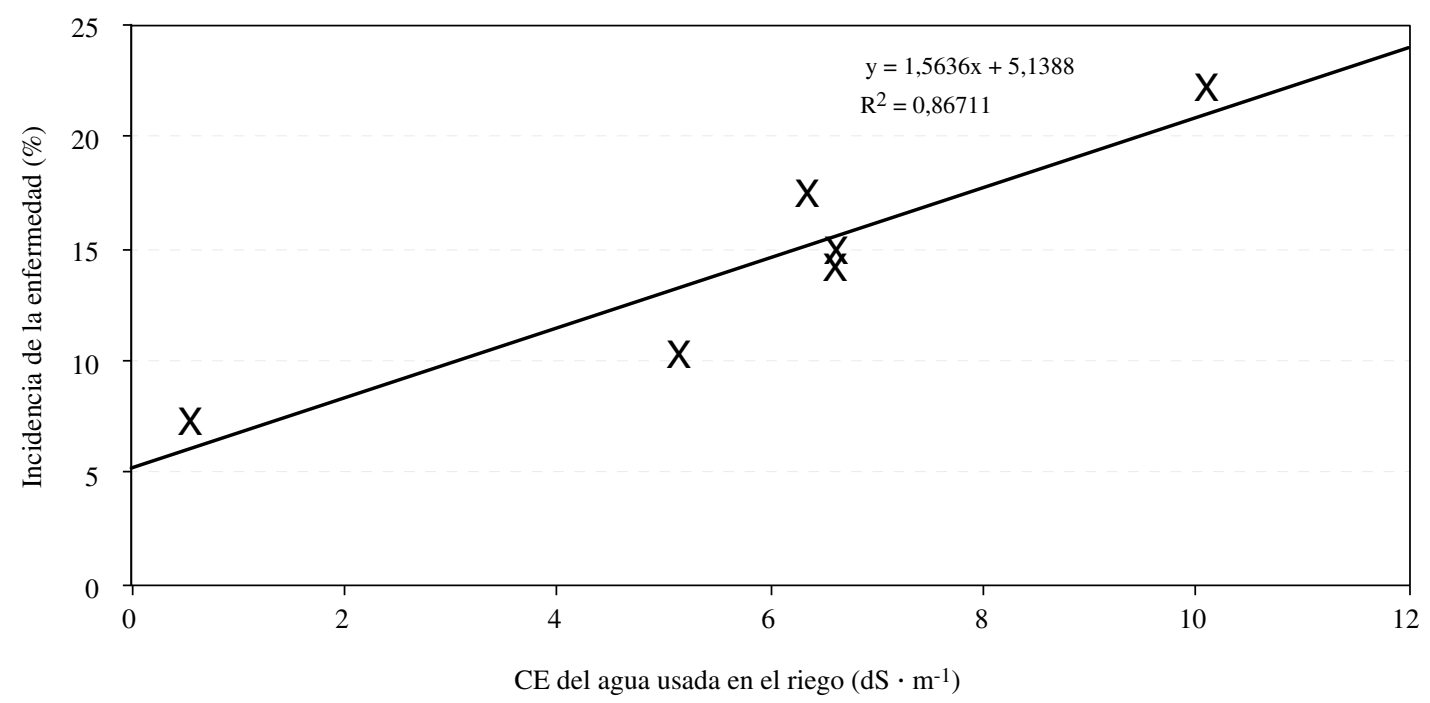

Figura 2. Relación entre CE del agua de riego e incidencia del síndrome en los invernaderos.

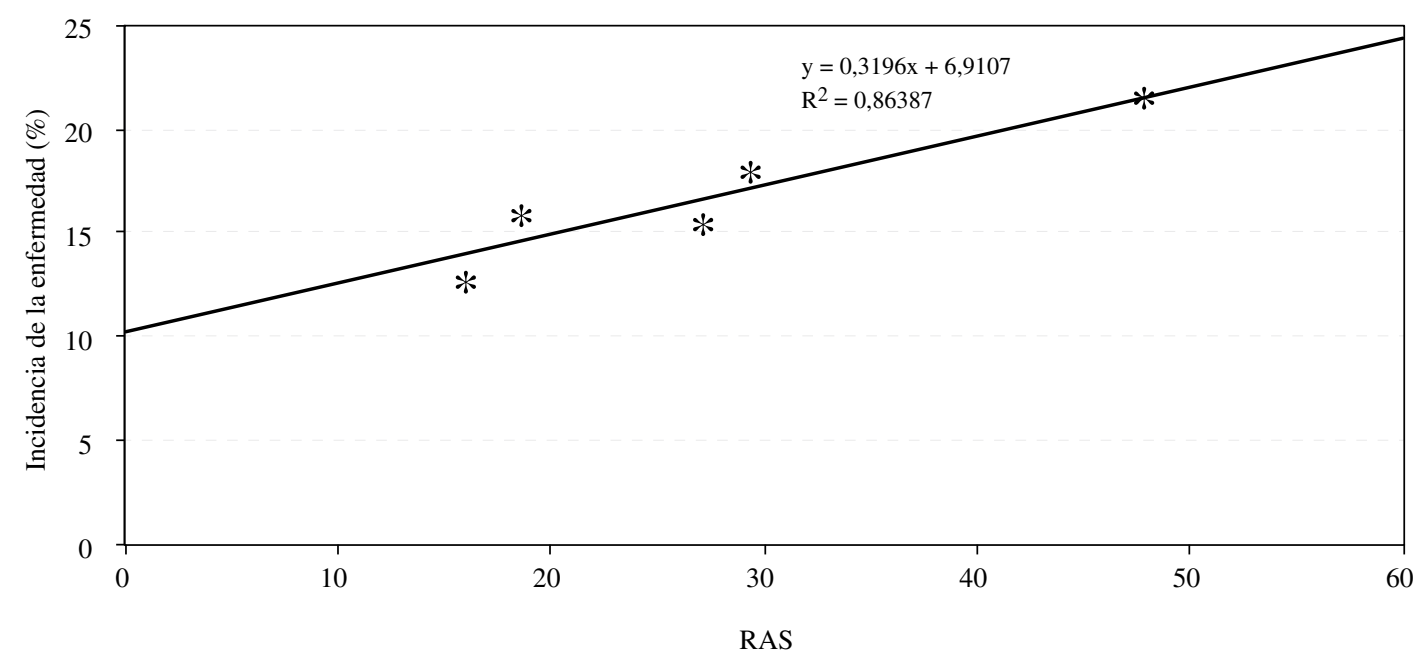

Figura 3. Relación entre SAR del agua de riego e incidencia del síndrome en los invernaderos estudiados. (Recta de regresión sin considerar al pozo 3 ).

representar un riesgo de sodificación. En la Figura 4 se representa la relación entre la incidencia del síndrome con las concentraciones de aniones $\mathrm{Cl}^{-}$. La correlación es todavía más clara $\left(\mathrm{R}^{2}=0,8943\right)$, donde parece existir casi una relación directamente proporcional. De nuevo, el pozo 3 es el que menor proporción presenta de $\mathrm{Cl}^{-}$, pero en proporción los $\mathrm{HCO}_{3}{ }^{-}$y los $\mathrm{CO}_{3}{ }^{=}$son mayores que en los otros pozos pudiendo aportar los cationes $\mathrm{Na}^{+}$. Finalmente, para el boro no ha podido establecerse una correlación con la gravedad del síndrome. Basta observar, para ello, los resultados presentados en la Tabla 4.

Un argumento adicional sobre la no causalidad de $F$. oxysporum podría establecerse a partir de la desinfección con metam-sodio. La desinfección que no hizo disminuir la expresión del síndrome, (el Inv1 del pozo 5), comparativamente, están más próximos a los invernaderos no desinfectados.

En el concepto de enfermedad establecido por Van der Plank (1963), donde el ambiente, el 
Tabla 4. Concentración de cationes $\left(\mathrm{Ca}^{++}, \mathrm{Mg}^{++}, \mathrm{Na}^{+}, \mathrm{K}^{+}, \mathrm{B}\right)$ en las aguas de los pozos utilizadas para regar.

\begin{tabular}{|c|c|c|c|c|c|c|c|c|c|}
\hline \multirow{2}{*}{ Muestra } & \multicolumn{2}{|c|}{ Calcio } & \multicolumn{2}{|c|}{ Magnesio } & \multicolumn{2}{|c|}{ Sodio } & \multicolumn{2}{|c|}{ Potasio } & \multirow{2}{*}{$\frac{\text { Boro }}{\mathrm{mg} \cdot \mathrm{L}^{-1}}$} \\
\hline & $\mathrm{mg} \cdot \mathrm{L}^{-1}$ & $\mathrm{meq} \cdot \mathrm{L}^{-1}$ & $\mathrm{mg} \cdot \mathrm{L}^{-1}$ & $\mathrm{meq} \cdot \mathrm{L}^{-1}$ & $\mathrm{mg} \cdot \mathrm{L}^{-1}$ & meq $\cdot \mathrm{L}^{-1}$ & $\mathrm{mg} \cdot \mathrm{L}^{-1}$ & $\mathrm{meq} \cdot \mathrm{L}^{-1} 1$ & \\
\hline P1 & 147 & 7,34 & 176 & 14,48 & 2235,83 & 97,25 & 20,91 & 0,53 & 0 \\
\hline $\mathrm{P} 2$ & 279 & 13,92 & 282 & 23,21 & 4735,08 & 205,96 & 15,41 & 0,39 & 0 \\
\hline P3 & 62 & 3,09 & 86 & 7,08 & 2498,91 & 108,7 & 31,93 & 0,82 & 0,5 \\
\hline P4 & 172 & 8,58 & 171 & 14,07 & 2104,29 & 91,53 & 26,42 & 0,68 & 14,1 \\
\hline P5 & 170 & 8,48 & 175 & 14,4 & 1446,6 & 62,92 & 20,91 & 0,53 & 6,4 \\
\hline P6 & 150 & 7,49 & 163 & 13,41 & 1183,52 & 51,48 & 9,9 & 0,25 & 0,8 \\
\hline
\end{tabular}

Tabla 5. Concentraciones de aniones (carbonatos, bicarbonatos, cloruros, sulfatos y nitratos) de las aguas de los pozos utilizadas para regar.

\begin{tabular}{lcllllllrrr}
\hline \multirow{2}{*}{ Muestra } & \multicolumn{2}{c}{$\mathrm{CO}_{3}{ }^{2=}$} & \multicolumn{2}{c}{$\mathrm{HCO}_{3}^{-}$} & \multicolumn{2}{c}{$\mathrm{Cl}^{-}$} & \multicolumn{2}{c}{$\mathrm{SO}_{4}{ }^{2=}$} & \multicolumn{2}{c}{$\mathrm{NO}_{3}^{-}$} \\
\cline { 2 - 11 } & $\mathrm{mg} \cdot \mathrm{L}^{-1}$ & $\mathrm{meq} \cdot \mathrm{L}^{-1}$ & $\mathrm{mg} \cdot \mathrm{L}^{-1}$ & $\mathrm{meq} \cdot \mathrm{L}^{-1}$ & $\mathrm{mg} \cdot \mathrm{L}^{-1}$ & $\mathrm{meq} \cdot \mathrm{L}^{-1}$ & $\mathrm{mg} \cdot \mathrm{L}^{-1}$ & $\mathrm{meq} \cdot \mathrm{L}^{-1}$ & $\mathrm{mg} \cdot \mathrm{L}^{-1}$ & $\mathrm{meq} \cdot \mathrm{L}^{-1}$ \\
\hline P1 & 31,8 & 1,06 & 116,54 & 1,91 & 1930,42 & 54,45 & 1086,41 & 11,31 & 158,84 & 2,56 \\
P2 & 0 & 0 & 290,44 & 4,76 & 3847,71 & 108,53 & 1158,55 & 12,06 & 33,94 & 0,55 \\
P3 & 61,81 & 2,06 & 132,4 & 2,17 & 515,13 & 14,53 & 465,98 & 4,85 & 31,96 & 0,52 \\
P4 & 60,01 & 2 & 78,71 & 1,29 & 1840,01 & 51,9 & 1653,92 & 17,22 & 238,13 & 3,84 \\
P5 & 47,41 & 1,58 & 185,49 & 3,04 & 1840,37 & 51,91 & 1548,12 & 16,12 & 231,05 & 3,73 \\
P6 & 78,61 & 2,62 & 195,25 & 3,2 & 1391,88 & 39,26 & 1432,69 & 14,91 & 41,21 & 0,66 \\
\hline
\end{tabular}

patógeno y el hospedador tienen el mismo peso en el desarrollo de la enfermedad, no se contempla el ambiente suelo. Se refiere esencialmente al ambiente aéreo, sin embargo, el ambiente suelo es determinante para la expresión de una enfermedad de origen edáfico. El suelo ha sido considerado como un "ente vivo", donde la interacción entre el hospedador y el patógeno está mediatizada por las interacciones de aquellos con la fracción viva y la físico-química (Tello Marquina et al., 2011). Por consiguiente, el trabajo presentado deberá ser interpretado desde esa óptica.

El tomate ha sido agrupado dentro de las especies vegetales moderadamente tolerantes a la salinidad, pudiendo ser afectado cuando la CE del agua de riego excede $2,5 \mathrm{dS} \cdot \mathrm{m}^{-1}$. Aguas con concentraciones altas de sales merman el desarrollo vegetativo pero incrementa el contenido en azúcares del fruto (Cuartero y Fernández Muñoz, 1999; Cuartero Zueco et al., 1995; Mass, 1986). En situaciones de alta salinidad en el agua de riego puede apreciarse disminución del tamaño de la planta (hojas, frutos, altura, diámetro del tallo), clorosis en las hojas y necrosis xilemática intensa, asociándose podredumbre apical en los frutos y necrosis epidérmicas en las partes bajas del tallo (Pellicer Botia et al., 1998). Triky-Dotan (2005) y Schoeneweiss (1975) sugieren
Tabla 6. pH, conductividad eléctrica y Ratio Adsorción de sodio de las aguas utilizadas para regar.

\begin{tabular}{lccc}
\hline Muestra & $\mathrm{pH}$ & $\mathrm{CE}\left(\mathrm{dS} \cdot \mathrm{m}^{-1}\right)$ & $\mathrm{SAR}$ \\
\hline P1 & 7,92 & 6,35 & 29,44 \\
P2 & 7,25 & 10,1 & 47,80 \\
P3 & 8,82 & 0,55 & 48,20 \\
P4 & 7,96 & 6,63 & 27,20 \\
P5 & 8,02 & 6,63 & 18,60 \\
P6 & 7,46 & 5,15 & 15,93 \\
\hline
\end{tabular}

que los efectos salinos sobre plantas de tomate son irreversibles, ya que no desaparecen al regar con agua no salina, además, sensibiliza a las plantas frente a la enfermedad conocida como podredumbre del cuello y de las raíces (agente causal Fusarium oxysporum f. sp. radicis-lycopersici). Es decir, la salinidad elevada ocasiona un síndrome descrito por diversos autores a lo largo de los años, y predispone al hospedador a contraer la enfermedad.

¿Qué pasa con los patógenos del suelo? En los casos estudiados suelen ser muy resistentes a la salinidad, especialmente a la originada por $\mathrm{NaCl}$. Así ha sido propuesto para Phytophthora por Blaker y Mac Donald (1985); Para Penicillium, Aspergillus, Fusarium por Tresner y Hayes (1971); 


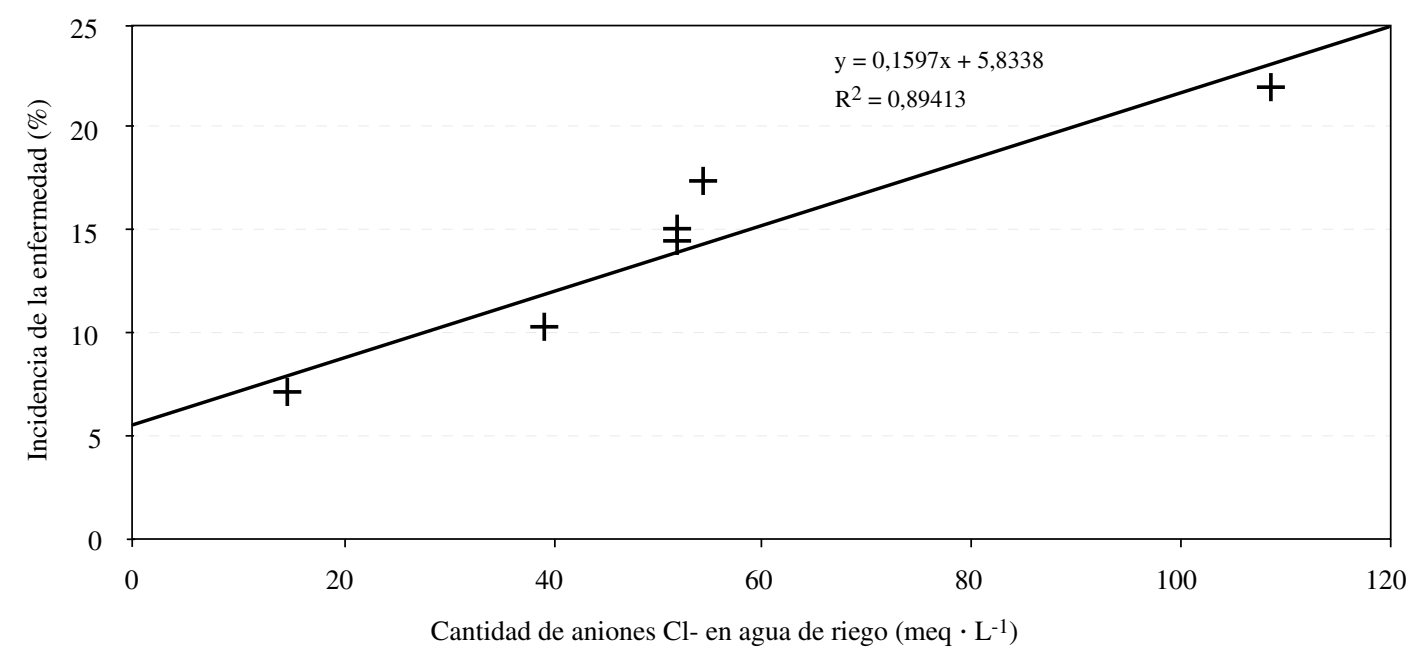

Figura 4. Relación entre cantidad de cloruros del agua de riego e incidencia del síndrome en los invernaderos estudiados.

para Fusarium oxysporum f. sp. vasinfectum (Ragazzi y Vecchio, 1992); para Fusarium culmorum (Palmero et al., 2009) y para F. oxysporum (Palmero et al., 2010), y además se han apreciado cambios en el comportamiento de estos hongos cuando se desarrollan en un medio con alta salinidad (Ragazzi et al., 1994; Palmero et al., 2010).

Si la salinidad en tomate origina un síndrome conocido y varios hongos patógenos del suelo resisten altas concentraciones salinas in vitro, parece procedente conocer cómo interaccionan estos dos factores en la expresión de las micosis de origen edáfico. Teniendo en cuenta la bibliografía consultada no podría decirse, como regla general, que la salinidad incrementa la gravedad de la enfermedad.

Varios trabajos sugieren que las fusariosis de las plantas pueden agravarse por efecto de los riegos con agua salina (Jones et al., 1993; Nachmias et al., 1993; Sivan y Chet, 1993; Woltz et al., 1992; TrikyDotan et al., 2005). Otros sugieren lo contrario, es decir, que la salinidad en el agua de riego disminuye la enfermedad (De la Perriere et al., 1995; Elmer, 1997, 2002, 2003; Elmer y La Mondia, 1999). El efecto sobre la expresión de otras enfermedades ha sido puesto de manifiesto para otros patógenos. Así, para Phytophthora (Swiecki y MacDonald, 1991); Rhizoctonia (Elmer y LaMondia, 1999); Verticillium dahliae y Alternaria solani (Nachmias et al., 1993 y Pellicer et al., 1998); Pythium (Rasmussen y Stanghellini, 1988).
La relación del $\mathrm{NaCl}$ con las enfermedades de las plantas, tanto si la salinización ocurre en el suelo como en el agua de riego, ha sido explicada de diferentes maneras.

Elmer y LaMondia (1999) y Elmer (2003), indagando sobre una práctica agrícola antigua en espárrago, consistente en añadir $\mathrm{NaCl}$ al suelo (560 a $1.200 \mathrm{~kg} \cdot \mathrm{ha}^{-1}$ ) para controlar las fusariosis del cuello y de las raíces del espárrago causadas por Fusarium proliferatum y Fusarium oxysporum, comprueban que la enfermedad disminuye y se incrementa la producción. Este hecho se ha relacionado con el incremento de cloruros $\left(\mathrm{Cl}^{-}\right)$, de manera que el $\mathrm{NaCl}$ tiene un efecto fungistático y no se descarta una influencia en la susceptibilidad del hospedador, comprobando una relación con la colonización de las raíces por las bacterias de los géneros Pseudomonas y Serratia, las que se relacionan con la disponibilidad del manganeso, contribuyendo así a la resistencia de las plantas a las enfermedades.

El incremento de la gravedad de la enfermedad por efecto de la salinidad con $\mathrm{NaCl}$ ha sido informado por diversos autores en el caso de la fusariosis del cuello y de las raíces del tomate (agente causal Fusarium oxysporum f. sp. radicis-lycopersici). Jones et al. (1993) propusieron que el abonado con $\left(\mathrm{NH}_{4}\right)_{2} \mathrm{SO}_{4}$ y el riego con agua salina $(\mathrm{NaCl}$, $2 \mathrm{~g} \cdot \mathrm{L}^{-1}$ ) y un $\mathrm{pH}$ ácido $(<4)$ en el suelo incrementaba la micosis, así como el alto contenido en $\mathrm{Na}$ y bajo en $\mathrm{Ca}$ se asociaron a una mayor gravedad de la 
enfermedad. Sin embargo, ciertas combinaciones de $\mathrm{CaCO}_{3}$, fuente nitrogenada y $\mathrm{NaCl}$, podían originar un alto grado de protección frente a la enfermedad. La relación con el nitrógeno fue abordada por Duffy y Defago (1999), quienes sugirieron que bajas concentraciones de $\mathrm{NH}_{4} \mathrm{NO}_{3}\left(39-79 \mathrm{mg} \cdot \mathrm{L}^{-1} \mathrm{~N}\right)$ reducían la gravedad de la enfermedad, pero concentraciones superiores a $100 \mathrm{mg} \cdot \mathrm{L}^{-1} \mathrm{de} \mathrm{N}$, la incrementaban significativamente. Tello Marquina (1984) sugiere que la salinidad podría tener un efecto en la expresión de la fusariosis vascular y la verticiliosis del tomate. Por su parte Triky-Dotan et al. (2005) sugieren que la salinidad del agua de riego con $\mathrm{NaCl}$ en cultivo en campo incrementa significativamente la fusariosis del cuello y de las raíces del tomate. De manera que $\mathrm{CE}$ de 3,2 a $0,4 \mathrm{dS} \cdot \mathrm{m}^{-1}$ originan aumentos del $75 \%$ y $38 \%$, respectivamente, en el trasplante. Dicho efecto, 250 días después del trasplante, incrementó la expresión de la enfermedad en $12 \%\left(\mathrm{CE}=4,6 \pm 0,1 \mathrm{dS} \cdot \mathrm{m}^{-1}\right)$ y un $4 \%\left(\mathrm{CE}=1,2 \pm 0,1 \mathrm{dS} \cdot \mathrm{m}^{-1}\right)$. Mientras el patógeno no es afectado por la salinidad, esta predispone a las plantas frente a la micosis.

\section{Conclusiones}

Los resultados revisados en este trabajo se refieren a una enfermedad de origen fúngico. La complejidad sobre el efecto de la salinidad abarca desde el efecto sobre el estado vegetativo de las plantas de tomate hasta la predisposición de estas a la enfermedad, pasando por dos situaciones contrapuestas: en unos casos aumentan las micosis $\mathrm{y}$ en otros disminuyen. Aspectos reveladores sobre las fuentes de fertilización y sobre la microbiología del suelo evidencian la complejidad del fenómeno de la salinidad y las enfermedades.

Los resultados presentados en este trabajo difieren de manera importante con lo sugerido por diversos autores. En primer lugar por el hecho de estar referidos a una prospección de invernaderos donde se estaban confundiendo los síntomas con dos enfermedades del tomate, una causada por Fusarium oxysporum f. sp. lycopersici y otra por Fusarium oxysporum f. sp. radicis-lycopersici. Las inoculaciones realizadas pusieron en evidencia que en las condiciones de ensayo esta causalidad no podía ser establecida. Así lo corroboraba, también, la falta de expresión de las desinfecciones del suelo con diferentes fumigantes. A partir de aquí, podría suponerse que la salinidad tenía una relación de causalidad sobre el síndrome observado. La salinidad del agua de riego era muy elevada, especialmente si se compara con la ensayada por los autores antes mencionados (Figuras 3, 4 y 5 y Tablas 4, 5 y 6), donde la salinidad podría ser la causa del síndrome. Sin embargo, ¿cómo explicar la presencia de Fusarium oxysporum en el xilema y las raíces necrosadas sin ser patógeno? ¿Podría instalarse un organismo no patógeno en el interior de las plantas por el efecto de la debilidad que les causaba el riego salino repetido a lo largo del cultivo y de los años?

En este sentido son reveladores los resultados presentados por Polizzi et al. (2007) y Dimartino et al. (2011). En condiciones de riego con agua salina el cultivo del tomate en sistemas sin suelo (en sustratos) permite a las bacterias Pseudomonas putida y Ps. fluorescens, reputados habitantes de la rizosfera de las plantas, expresarse como enfermedad en pleno cultivo, originando la muerte de las plantas con un síntoma fundamental: necrosis del xilema y de la médula. La salinidad fue producida por $\mathrm{NaCl}$ (31 meq. $\mathrm{L}^{-1} \mathrm{de} \mathrm{Cl}^{-}$y $31 \mathrm{meq} \cdot \mathrm{L}^{-1} \mathrm{de} \mathrm{Na}^{+}$), que daban lugar a una CE de 4,50 dS $\cdot \mathrm{cm}^{-1}$. Esta situación es llamativa por novedosa y, especialmente, porque $P S$. putida y Ps. fluorescens son reputados agentes de control biológico. ¿Podría compararse esta situación con el síndrome presentado en este trabajo? Lo que es cierto es que los trabajos de Polizzi et al. (2007) y Dimartino et al. (2011) abren un camino de investigación hasta el presente no explorado.

\section{Literatura Citada}

Ayers, R.S. and Wescot, D.W.

1985. Calidad del agua de riego para la agricultura. Estudio F.A.O. Roma. 174 pp.

Blaker, N.S. and Macdonald, J.D.

1986. The role of salinity in the development of Phytophthora root-rot of citrus. Phytopathology 76 (10): 970-975.
Cuartero, J. and Fernández-Muñoz, R. 1999. Tomato and salinity. Scientia Horticulturae 78 (1-4): 83-125.

Cuartero Zueco, J.; Fernández-Muñoz, R. y González-Fernández, J.J. 1995. Estreses abióticos. En: El cultivo del tomate. 352-383. Ediciones Mundi-Prensa. Madrid. 
De la Perriere, R.A.; Amir, H. and Bounaga, N.

1995. Prospects for integrated control of bayoud' (fusarium wilt of the date palm) in algerian plantations. Crop Protection 14 (3): 227-235.

Delegación Provincial de Agricultura de la Junta de Andalucía en Almería.

Memorias resumen de los años 2002 a 2011, 2-4.

Dimartino, M.; Panebianco, S.; Vitale, A.; Castello, I.; Leonardi,

C.; Cirvilleri, G. and Polizzi, G.

2011. Occurrence and pathogenicity of Pseudomonas fluorescens and P. putida on tomato plants in Italy. J Plant Pathol 93 (1):79-87.

Duffy, B.K. and Defago, G.

1999. Macro and microelement fertilizers influence the severity of fusarium crown and root rot of tomato in a soilless production system. HortScience 34 (2): 287-291.

Elmer, W.H.

1997. Influence of chloride and nitrogen form on Rhizoctonia root and crown rot of table beets. Plant Dis. 81 (6): 635-640.

Elmer, W.H.

2002. Influence of inoculum density of Fusarium oxysporum f. sp cyclaminis and sodium chloride on cyclamen and the development of Fusarium wilt. Plant Dis. 86 (4): 389-393.

Elmer, W.H.

2003. Local and systemic effects of $\mathrm{NaCl}$ on root composition, rhizobacteria, and fusarium crown and root rot of asparagus. Phytopathology 93 (2): 186-192.

Elmer, W.H. and LaMondia, J.A.

1999. Studies on the suppression of Fusarium crown and root rot of asparagus with $\mathrm{NaCl}$. Ixth International Asparagus Symposium (479): 211-217.

Gerlach, W. and Nirenberg, $\mathrm{H}$.

1982. The genus Fusarium: A pictorial atlas. Kommissionsverlag P. Parey, Berlin. 406 pp.

Jones, J.P.; Jones, J.B.; Woltz, S.S. and Scott, J.W.

1993. Influence of soil-ph, nitrogen-source, and transplant drenches on development of crown rot of tomato. Proceedings of the 106th Annual Meeting of the Florida State Horticultural Society $106: 170-172$.

Komada, H.

1975. Development of a selective medium for quantitative isolation of Fusarium oxysporum from natural soil. Review of Plant Protection Research 8: 114-125.

Mass, E.V.

1986. Salt tolerance of plant. Appl. Agric. Res. 1: 12-26.

Nachmias, A.; Kaufman, Z.; Livescu, L.; Tsror, L.; Meiri, A. and Caligari, P.D.S.

1993. Effects of salinity and its interactions with disease incidence on potatoes grown in hot climates. Phytoparasitica 21 (3): 245-255.

Nelson, P.E.; Toussoun, T.A. and Marassas, W.F.O.

1983. Fusarium Species and Illustrated Manual for Identification. The Pennsylvania State University Press, University Park, and London. 193 pp.

Palmero, D.; De Cara, M.; Iglesias, C. and Tello, J.C.

2009. The interactive effects of temperature and osmotic potential on the growth of aquatic isolates of Fusarium culmorum. Geomicrobiol J. 26 (5): 321-325.

Palmero, D.; De Cara, M.; María Moreno, M.; Iglesias, C. and César Tello, J.

2010. Stimulation of mycelial growth of pathogenic and seabed isolates of Fusarium oxysporum in presence of salts. African Journal of Microbiology Research 4(17): 1859-1861.
Pellicer Botia C.; Balsalobre Balibrea E.; Rincón Sánchez L. and Sáez Sironi J.

1998. Deficiencias nutricionales y otras alteraciones fisiológicas. En: La sanidad del tomate. Ed. Phytoma España. S.L. Valencia., 255-283.

Polizzi, G.; Diamartino, M.A.; Panebianco, S. and Cirvilleri, G. 2007. A new emergence on soilless tomato cultures in Sicily vascular and pith discolouration caused by Pseudomonas fluorescens and P. putida. Journal of plant Pathology 89: 54-55.

Porta, A.J.; López-Acebedo, M. y Roquero, C.

2003. Edafología para la agricultura y el medio ambiente. Ed. Mundi-Prensa. Madrid. 849 pp.

Ragazzi, A. and Vechio, V.

1992. Behaviour of chlamydospore of Fusarium oxysporum f. sp. vasinfectum in substrates containing sodium chloride. Phytopathol. Mediterr. 31: 85-87.

Ragazzi, A.; Vechio, V.; Dellavalle, I.; Cucchi, A. and Mancini, F. 1994. Variations in the pathogenicity of Fusarium oxysporum f.sp. vasinfectum in relation to the salinity of the nutrient medium. Zeitschrift Fur Pflanzenkrankheiten Und Pflanzenschutz-Journal of Plant Diseases and Protection 101 (3): 263-266.

Rasmussen, S.L. and Stanghellini, M.E.

1988. Effect of salinity stress on development of Pythium blight in agrostis-palustris. Phytopathology 78 (11): 1495-1497.

Schoeneweiss, D.F.

1975. Predisposition, stress, and plant disease. Аnnu Rev Phytopathol. 13: 193-211.

Segura Visiedo, J.M.

2005. Evaluación de alternativas al bromuro de metilo en cultivo enarenado de tomate, bajo invernadero y abrigo de malla en Pujaire (término municipal de Nijar, Almería). Proyecto fin de carrera. Universidad de Almería. 114 pp.

Sivan, A. and Chet, I.

1993. Integrated control of Fusarium crown and root-rot of tomato with Trichoderma harzianum in combination with methyl-bromide or soil solarization. Crop Protection 12 (5): 380-386.

Swiecki, T.J. and Macdonald, J.D.

1991. Soil-salinity enhances Phytophthora root-rot of tomato but hinders asexual reproduction by Phytophthora parasitica. J Am Soc Hort Sci. 116 (3): 471-477.

Tello, J.; Varés, F. y Lacasa, A.

1991. Análisis de muestras. En: Manual de laboratorio. Diagnóstico de hongos, Bacterias y Nematodos fitopatógenos. Ed: Ministerio de Agricultura, Pesca y Alimentación. Madrid. 39-77.

Tello Marquina, J.C.

1984. Enfermedades criptogámicas en hortalizas. Comunicaciones INIA. Serie: Protección Vegetal $\mathrm{N}^{\mathrm{o}} 22$, $342 \mathrm{pp}$.

Tello Marquina, J.C.; De Cara García, M.; Moreno Díaz, A.; Santos Hernández, M. y Palmero Llamas, D.

2011. El suelo como "ente vivo" y su relación con las enfermedades de las plantas. In: Agricultura Ecológica en Secano. Ed. Ministerio de Medio Ambiente y Medio Rural y Marino, Junta de Comunidades de Castilla-La Mancha, Sociedad Española de Agricultura Ecológica y Ediciones Mundi-Prensa. 181-220.

Tello Marquina, J.C. y Lacasa Plasencia, A.

1990. Fusarium oxysporum en los cultivos intensivos del litoral mediterráneo de España. Fases parasitaria (Fusariosis 
vasculares del tomate y del clavel) y no parasitaria. Boletín de Sanidad Vegetal (fuera de serie) 19: 1-185.

Tresner, H.D. and Hayes, J.A.

1971. Sodium chloride tolerance of terrestrial fungi. Appl Microbiol. 22 (2): 210-213.

Triky-Dotan, S.; Yermiyahu, U.; Katan, J. and Gamliel, A. 2005. Development of crown and root rot disease of tomato under irrigation with saline water. Phytopathology 95 (12): 1438-1444.
Van der Plank, J.E.

1963. Disease Resistance in Plants. Ed: Academic Press Inc. New-York y London. 349 pp.

Woltz, S.S.; Jones, J.P. and Scott, J.W.

1992. Sodium-chloride, nitrogen-source, and lime influence Fusarium crown rot severity in tomato. HortScience, 27 (10): 1087-1088. 
